\title{
Evaluation of A Pool of Biomimetic Peptides on Human Hair Follicles: A Preclinical Study
}

\section{Rodella Luigi Fabrizioo $^{1,2}$, Rezzani Rita ${ }^{1,2}$, Marzani Barbara ${ }^{3}$, Pinto Daniela $^{3}$, Cocchi Marco ${ }^{1}$ Sorbellini Elisabetta ${ }^{4}$, and Rinaldi Fabio ${ }^{*}$}

${ }^{1}$ Department of Clinical and Experimental Sciences, Division of Anatomy and Physiopathology, University of Brescia, Viale Europa 11, 25123 Brescia, Italy

${ }^{2}$ Interdipartimental University Center of Research "Adaption and Regeneration of Tissues and Organs - (ARTO)", University of Brescia, Viale Europa 11, 25123 Brescia, Italy

${ }^{3}$ Giuliani S.p.A., Via Palagi 2, 20129, Milan, Italy

${ }^{4}$ International Hair Research Foundation, Milan, Italy

fabio.rinaldi@studiorinaldi.com

*Corresponding Author: Rinaldi Fabio, International Hair Research Foundation, Milan, Italy.

Abstract

Background: Biomimetic peptides are synthetic agonists of naturally occurring growth factors and completely mimics the action of the parental molecules. They offer a great opportunity in cosmetic and dermatological applications. Since on the marketplace there are many biomimetic peptides and product containing them in association, but, unfortunately, only few peptides have strong scientifically published evidence about their own claim, we investigated the effect of a mix of octapeptide-2 (0.05\%), copper tripeptide-1 (0.05\%), oligopeptide20 (0.5\%), acetyl decapeptide-3 (0.5\%) (mix of peptides: MGF) in human hair follicle (HFs) from AGA subjects.

Materials and Methods: 400 HFs from 10 subjects affected by AGA were cultivated in 4 different conditions: supplemented William's E culture medium (NM) with or without MGF; William's E culture medium without supplements (WN) with MGF presence or not. HFs elongation, staging and hair cycle score measurements were taken at 24-hour intervals until the 96th hour of cultivation. The levels of Ki-67, a marker of cellular proliferation, were determined immunohistochemically on the HFs in different condition: no medium (dried), Ringer Lactate (RL), PRP, and MGF.

Results: MGF added to NM or WNM medium induced a higher hair shaft elongation in comparison with media alone. The stimulation of hair shaft was statistically significant when measured at $96 \mathrm{~h}$ of treatment with MGF supplementation to the culture media $(p<0.05, W M+M G F s$ vs WM and $p<0.01, N M+M G F s$ vs NM).

Culture of HFs in the presence of MGF promoted hair growth anagen phase, as showed by hair cycle score decrease $(p<0.05, W M+M G F$ vs $W M$ and $N M+M G F$ vs $N M)$.

The immune fluorescence staining for Ki-67 revealed that from 4 hours up to 8 hours of treatment there was a statistically significant preservation of proliferative activity by PRP and MGF (T4 $h$ : PRP vs RL $p<0.05$; PRP vs Dried $p<0.01 ;$ MGF vs Dried $p<0.001$; MGF vs RL $p<0.01$; T8 h: PRP vs $R L p<0.001$; PRP vs Dried $p<0.0001$; MGF vs Dried $p<0.0001$; MGF vs RL $p<0.0001$ ).

Conclusions: In this preclinical study, MGF can be considered a useful therapeutic agent to stimulate hair growth by promoting anagen phase and cell proliferation.

Keywords: Biomimetic peptides; Human hair follicle; Hair growth; octapeptide-2; copper tripeptide-1; oligopeptide-20, acetyl decapeptide-3. 
Evaluation of A Pool of Biomimetic Peptides on Human Hair Follicles: A Preclinical Study

\section{INTRODUCTION}

Peptides are short chain of amino acids linked by peptide bonds and in term of chemical complexity they are in the middle between small molecules and larger proteins $(1,2)$. The interest in peptide arose from the pioneer research by du Vigneau and Tuppy with the isolation of the first peptide hormone identifying it as cyclic octapeptide, later named oxytocin (3). Starting from this study many progresses were made and hundreds of peptides, with variable sequence length, have been identified. These peptides have several roles in biological and pathological processes. In particular they show important and different roles in growth, defense, immunity and homeostasis $(2,4)$. With regard to skin and dermatological applications, oligopeptides (short sequence consisting of 2-20 amino acids) represent a very promising application (5). The dermatological era of bioactive peptides, also called biomimetic peptides, began from the wound healing studies (6) and nowadays is of the major interest.

These peptides have been designed mainly to target different mechanisms to improve skin appearance and delay skin aging processes and only few peptides were studies for hair growth targeting.

The first peptide identified for the hair growth is GHK$\mathrm{Cu}$ peptide, a copper complex of a glycyl-L-histidyl-Llysine peptide. This peptide is able to activate many remodeling related processes and one of particular interest is the anti-inflammatory effects. It suppress free radicals, thromboxane formation, release of oxidizing iron, transforming growth factor beta-1, tumor necrosis factor alpha and protein glycation. Moreover the peptide increases superoxide dismutase and vessel vasodilation, blocking also ultraviolet damage to skin keratinocytes and improving fibroblast recovery after X-ray treatments (7).

In particular, GHK-Cu is able to counteract hair loss through the stimulation of stem cells increasing hair follicle size (7). L-alanyl-L-histidyl-L-lysine-Cu2+ (AHK-Cu) peptide is one of the tripeptide-copper complex. Study by Pyo and collaborators (8) on ex-vivo human hair follicle demonstrated that hair growth stimulation by AHK-Cu is due to the ability in promoting dermal papilla cells proliferation, reducing the apoptosis.

The peptide copper complex ALAVAX is composed 5-aminolevulinic acid (5-ALA) and GHK complex.
ALAVAX was developed to improve 5-ALA drawbacks adding the efficacy of GHK in hair loss.

A double blind randomized clinical trial on men suffering of hair loss (type II-V Norwood-Hamilton classification) with 6 months of topical application for 6 months confirm the efficacy and the good tolerability of the treatment(9).

Biomimetic peptide is a synthetic agonist of naturally occurring growth factors and completely mimics the action of the parental molecules, consists of oligopeptides up to 15 amino acids and can provide clinical benefits similar to recombinant growth factors, reduce costs, and have greater chemical stability (10).

Octapeptide-2 is a synthetic peptide composed of 8 amino acid sequence (glutamic acid, glutamine, leucine, lysine, and threonine).

It is like of thymosin beta 4 growth factor, that is well known to stimulate hair growth by increasing angiogenesis and promoting the migration of stem cells and their immediate progeny to the base of the follicle, differentiation, and extracellular matrix remodeling $(11,12)$.

The octapeptide is able to increase keratinocyte and fibroblast growth in vitro and to avoid the degradation by peptidases and proteases the peptide is protected by micro-in capsulation.

Another interesting peptide is Decapeptide P3 (95-VGIGFHLQIY-104) selected between various peptides having partial sequence of FGF- 5 and FGF5S to antagonize FGF-5 to increase hair growth. In vitro assay showed that decapeptide P3 suppress FGF-5-driving proliferation in fibroblast cell lines by interaction with FGF-1 and FGF-2 receptors. The results were confirmedin vivo, on dorsal depilated mice, with an effect in hair pigmentation and hair follicle lengths (13).

Sh-polypeptide 9 is a VEGF-like synthetic biomimetic peptide (165 aa) in double-layered capsulation technology for improve skin penetration, stability and purity of the peptide.

In the study by Bassino et al., (14) the peptide activity was compared with Minoxidil in human microvascular endothelial cells (HMVEC) coculture with follicle dermal papilla cells (FDPC).

Sh-polypeptide 9 showed a higher activity at lower dose 
compared to Minoxidil in: increasing VEGF, stimulating the bFGF release and b-catenin production. Moreover Sh-polypeptide 9 is able to protect endothelium from oxidative stress by reduction of IL-1a, a negative hair growth regulator.

Most of peptides target skin firmness and nevertheless the initial interest only few in targeting hair growth even if recently they are of great interest.

With the exception of the study reported by Schager (15) about the efficacy of a combination of 2 peptides (acetylhexapeptide-3 and pentapeptide-18) in a clinical study, no other evidence about associations are available, or, at least in scientific publication.

Because many cosmetic products on the marketplace are a mix of different peptides with different claims it should be desirable to have an evidence of the product efficacy in controlled studies.

Nowadays the peptides are important components in cosmetic formulation, due to their biological activities, but, to our knowledge, no studies were reported about their associations.

The aim of this study was to evaluate the efficacy of biomimetic peptide complex with important role in hair cycle regulation: octapeptide-2, copper tripeptide-1, oligopeptide-20, acetyl decapeptide-3 (MGF).

\section{MATERIALS AND MethodS}

\section{Isolation and Culture of Human Hair Follicles}

Human hair follicle were obtained during autologous hair transplantation surgery (by FUE technique) in AGA (II - IV grade of Hamilton scale), patients after informed consent. A total of 400 anagen HFs were obtained from 10 different individuals. No subject had any systemic diseases and no subject had received any pharmacological or cosmetic trichological treatment in the six months preceding the study.

Briefly, after separation of the epidermis and dermis from the dermosubcutaneous interface, anagen hair follicles were isolated from subcutaneous fat under a light microscope using watchmaker forceps and maintained in Williams E medium (SigmaAldrich) supplemented with $10 \mu \mathrm{g} / \mathrm{mL}$ insulin (Sigma-Aldrich), $10 \mathrm{ng} / \mathrm{mL}$ hydrocortisone (SigmaAldrich), 2mM L-glutamine (Invitrogen), $100 \mathrm{IU} / \mathrm{mL}$ penicillin (Invitrogen), and $100 \mu \mathrm{g} / \mathrm{mL}$ streptomycin (Invitrogen).
Hair follicles were maintained free-floating at $37^{\circ} \mathrm{C}$ in an atmosphere of 5\% CO2 and 95\% air in a humidified incubator. Hair follicles were cultivated for $0,24,48$, 72,96 hours in 4 different culture media, with the different active principles. For each subject 1) $10 \mathrm{HFs}$ were cultivated in supplemented William's E culture medium (standard medium- NM); 2) 10 HFs were cultivated in William's E culture medium without supplements (WN); 3) $10 \mathrm{HFs}$ were cultivated in supplemented William's E culture medium adding polypeptide MGF (250 $\mu \mathrm{l}$ : $0.05 \%$ octapeptide-2, $0.05 \%$ copper tripeptide-1, 0.5\% oligopeptide-20, 0.5\% acetyl decapeptide-3): 4) 10 HFs were cultivated in William's E culture medium WM with the addition of polypeptide MGF $(250 \mu \mathrm{l})$.

Another set of HFs were obtained from donor (occipital) area from 15 subjects underwent to autologous transplantation (10-8 HFs/subject). Immediately after transplantation HFs underwent to different condition: a) dried (no medium, exposed to room condition), b) Ringer Lactate (RL), c) Platelet Rich Plasma (PRP was obtained from each single donor after centrifugation, 17), and d) MGF. Proliferation evaluations were performed at T0, after 2 hours (T2 h), after 4 and 8 hours (respectively T4 h and T8 h).

\section{Measurement of Hair Follicle Length,} Morphology and Proliferation

Hair follicle length was defined as the entire length from the base of the hair follicle bulb to the tip of the hair shaft using a measuring scale attached to a microscope objective lens (Olympus, Hamburg, Germany). The digital images of the bulbs were analyzed through a specific image analyzer (Image Pro-Plus 4.5.1; Immagini e Computer, Milan, Italy).

Measurements were taken at 24-hour intervals until the 96th hour of cultivation.

Moreover, hair follicles of each group were staged and scored as follows: anagen VI, 100; early catagen, 200; mid-catagen, 300; late catagen, 400 (16).

The levels of Ki-67, a marker of cellular proliferation, were determined immuno histochemically as described previously (17) on HFs obtained by donor area from 15 subjects after treatment with different conditions.

\section{Statistical Analysis}

The data obtained were evaluated with Bonferroni corrected analysis of variance (Anova Test). $\mathrm{P}<0.05$ was considered significant. 


\section{RESULTS}

In Figure 1 are reported the data obtained from the HF culture of all subjects.

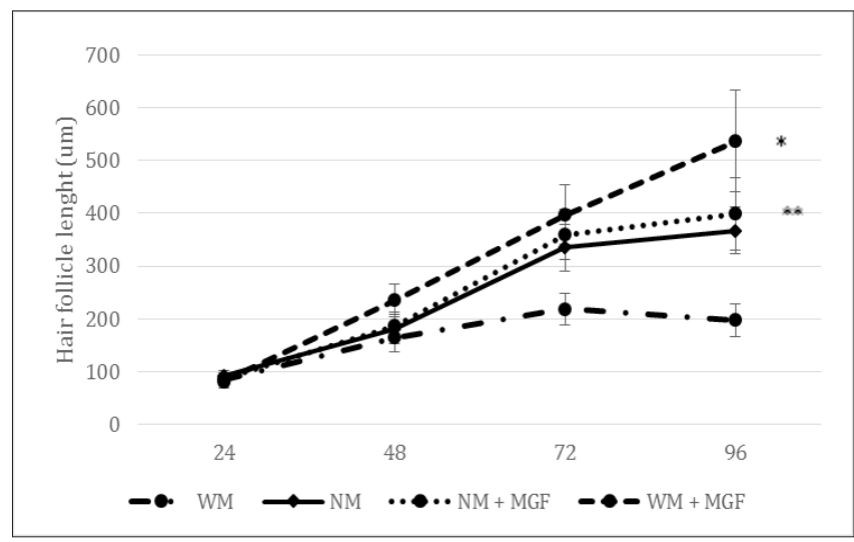

Figure 1. Quantitative analysis of hair growth after treatment with different medium. Hair follicle length was measured at 24-hour intervals. Data are based on 10 hair follicles/subjects/group and are reported as a mean \pm SEM.

${ }^{*} \mathrm{p}<0.05, \mathrm{WM}+\mathrm{MGFs}$ vs $\mathrm{WM}^{* *} \mathrm{p}<0.01, \mathrm{NM}+\mathrm{MGFs}$ vs NM.

A lengthening of the cultivated HF's shafts can be observed in all the four culture media.

In particular, the elongation (lengthening of shafts) in the medium NM, William's E with supplementation factors is significant, in accordance with literature data. In this case, the medium and the supplementation factors maintain the anagen phase up to 96 hours, thus enabling a constant elongation.

The growth of shafts in the medium WM, William's $\mathrm{E}$ without supplementation factors is detectable, although poor, thus showing a reduced maintenance of the anagen phase and therefore an insufficient capability of stimulating the elongation.

The HFs cultivation in the William's E NM medium with the addition of polypeptide MGFs induced a significantly elongation increase $(+31.64 \%)$ vs. the HFs cultivated in NM.

The growth of shafts in the medium William's E WM with the addition of polypeptide MGFs has a significant trend versus the WM. After 48 hours the growth is not different. It then continues on a linear basis until at 96 hours. The elongation difference is $51.85 \%$ higher in the shafts cultivated in NM at 96 hours $(39.18 \%$ at 72 hours).

Effects of MGF was investigated in hair cycling score (Figure 2). After 4 days of organ culture MGF was able to decrease hair cycle score values increasing the viability (i.e. anagen) of HFs in a statistically significant way $(\mathrm{p}<0.05)$ when compared to culture medium WM or NM.

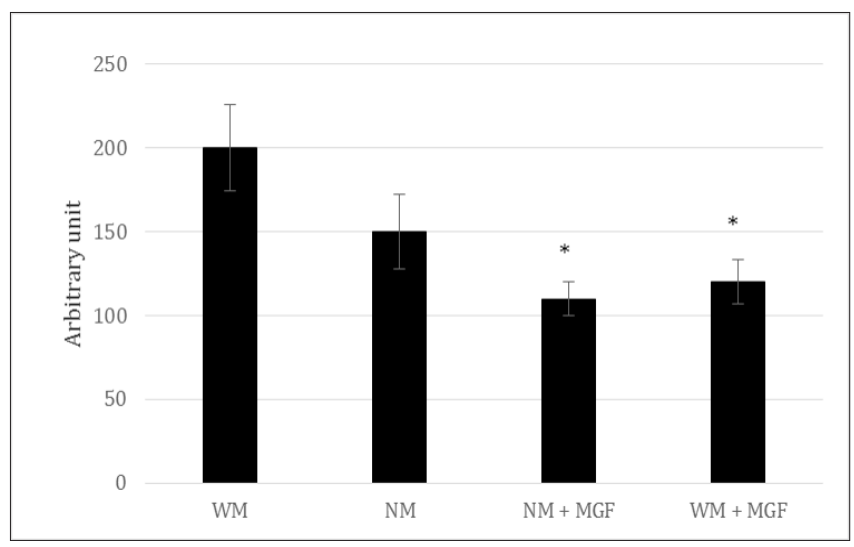

Figure 2. Hair cycle scores after treatment with OML extracts. (A) Hair follicles of each group were staged and scored as follows: anagen VI, 100; early catagen, 200; mid-catagen, 300; late catagen, 400. Data are based on 10 hair follicles per group and are reported as a mean \pm SEM. 
${ }^{*} \mathrm{p}<0.05, \mathrm{WM}+\mathrm{MGF}$ vs WM and NM + MGF vs NM.

Because anagen phase is supported by proliferative keratinocyte activities, we investigated the effect of MGF in organ cultures. The immune fluorescence staining for Ki-67 revealed that all the conditions induce a significant decrease of HF cell proliferation during the time course. The treatments did not influence the physiological cellular decay after 2 hours, but starting from 4 hours up to 8 hours of treatment there was a statistically significant preservation of proliferative activity by PRP and MGF (T4 h: PRP vs RL $\mathrm{p}<0.05$; PRP vs Dried $\mathrm{p}<0.01$; MGF vs Dried $\mathrm{p}<0.001$; MGF vs RL $\mathrm{p}<0.01$; T8 h: PRP vs RL $\mathrm{p}<0.001$; PRP vs Dried $\mathrm{p}<0.0001$; MGF vs Dried $\mathrm{p}<0.0001$; MGF vs RL $\mathrm{p}<0.0001)$.

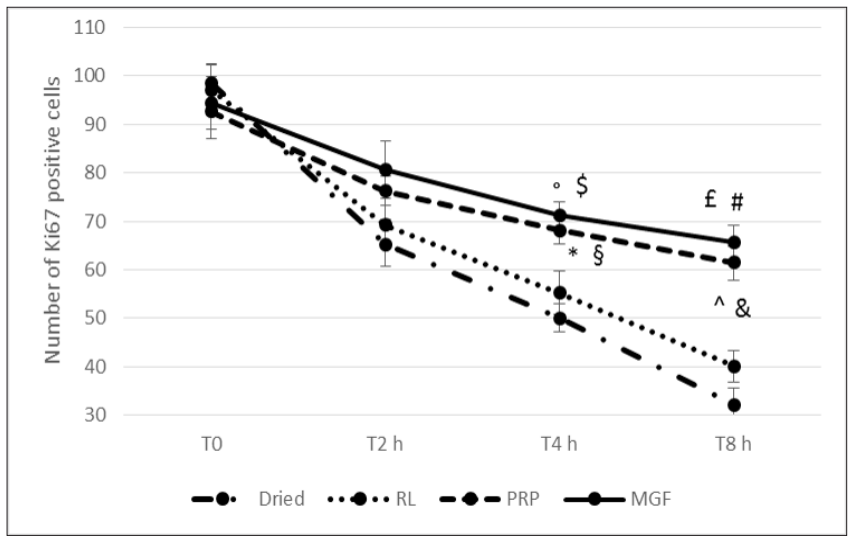

Figure 3. Number of Ki67 positive cell in HFs after treatment with different condition: dried (no medium, exposed to room condition), Ringer Lactate (RL), PRP, and MGF. Analysis were performed at T0, after 2 hours (T2 h), after 4 and 8 hours (respectively T4 $h$ and T8 h). Data are based on 8 hair follicles/subjects/group and are reported as a mean \pm SEM.

T4 h: * p <0.05 PRP vs RL; § $\mathrm{p}<0.01$ PRP vs Dried; ${ }^{\circ}$ $\mathrm{p}<0.001$ MGF vs Dried; $\$ \mathrm{p}<0.01 \mathrm{MGF}$ vs RL; T8 h: $\& \mathrm{p}<0.001$ PRP vs RL; ${ }^{\wedge} \mathrm{p}<0.0001$ PRP vs Dried; $€$ $\mathrm{p}<0.0001$ MGF vs Dried; \# $\mathrm{p}<0.0001$ MGF vs RL.

\section{Discussion AND CoNCLUSION}

Bioactive peptides in dermatology are a category of therapeutic aids in rapidly expansion.

They can target many physiological processes such as cell proliferation, protein synthesis, inflammation and pigmentation.

Cosmetic formulation containing biological active ingredients, such as peptides, in the last period find an important application not only in consumer skin care but also in dermatological field.

As reported by differentauthors peptides are the future for the possibility to provide an extraordinary range of active ingredients and the technology platform able to target specific skin conditions (18).

However the peptides applications are mainly addressed to the anti aging skin care, in particular in wrinkle-smoothing claim, despite a wide range of targets and applications.
Lot of peptides on marketplace unfortunately lack of in vitro robust evidences and in use test on volunteers with finish products.

The accessibility of these data, when present, is another important point to be evaluated. Many information are available only in the promotional/technical brochures, or commercial web-based advertising.

The data of the in vitro culture of 400 HFs obtained from the removal of hairs in an autologous transplant from 10 healthy volunteers show that the addition of polypeptide MGFs to the medium William's E with supplementation factors results in a significantly increased growth of shafts $(+31.64 \%$, $p>0.05)$ versus the supplemented medium NM.

The addition of polypeptide MGFs to the medium William's E WM results in an extremely significant increased growth of cultivated bulb shafts versus the medium WM $(51.85 \%, p>0.01)$. The growth of the bulbs is the same as $(0.13 \%)$ the growth in the medium NM without MGF. These data show the capability of MGF of stimulating the growth of bulbs even in a medium without supplementation factors, with a decrease in hair cycle score. 
Because cell proliferation is a very important event for anagen hair growth (19), in the second set of experiment, we investigate the proliferative effect of MGF in comparison with Ringer Lactate and PRP. To our knowledge, for the first time, it was demonstrated, in vitro, that a selected mix of biomimetic peptides (MGF) displayed a proliferative activity comparable to PRP treatment.

In conclusion, this study showed, in organ culture, the capacity of MGF to promote hair growth and, therefore, MGF can be useful as a therapeutic agent to enhance hair growth or slow progression of hair loss. Further studies are necessary in order to investigate the MGF effect in human controlled clinical trials.

\section{REFERENCES}

[1] Reddy B, Jow T, Hantash BM. Bioactive oligopeptides in dermatology: Part I. ExpDermatol 2012;21(8):563-8. doi: 10.1111/ j.1600-0625.2012.01528.x.

[2] Uhlig T, Kyprianou T, Martinelli FG, Oppici CA, Heiligers D, Hills D, et al. The emergence of peptides in the pharmaceutical business: From exploration to exploitation. EuPA Open Proteom2014;4:58-69. doi: 10.1016/j. euprot.2014.05.003

[3] Lintner K, Peschard O. Biologically active peptides: from a laboratory bench curiosity to a functional skin care product. Int J Cosmet Sci. 2000 Jun;22(3):207-18. doi: 10.1046/j.14672494.2000.00010.x.

[4] Hancock RE, Sahl HG. Antimicrobial and host-defense peptides as new anti-infective therapeutic strategies. NatBiotechnol. 2006 Dec;24(12):1551-7. doi: 10.1038/nbt1267

[5] Reddy B, Jow T, Hantash BM. Bioactive oligopeptidesindermatology: PartI.ExpDermatol. 2012 Aug;21(8):563-8. doi: 10.1111/j.16000625.2012.01528.x.

[6] Farrar GE Jr, Krosnick A. Milestones in clinical pharmacology. Wound healing. ClinTher. 1991 May-Jun;13(3):430-4.

[7] Pickart L. The human tri-peptide GHK and tissue remodeling. J Biomater SciPolym Ed. 2008;19(8):969-88. doi: $10.1163 / 156856208784909435$.
[8] Pyo HK, Yoo HG, Won CH, Lee SH, Kang YJ, Eun $\mathrm{HC}$, Cho KH, Kim KH. The effect of tripeptidecopper complex on human hair growth in vitro. ArchPharm Res. 2007 Jul;30(7):834-9.

[9] Lee WJ, Sim HB, Jang YH, Lee S-J, Kim DW, Yim S-H. Efficacy of a Complex of 5-Aminolevulinic Acid and Glycyl-Histidyl-Lysine Peptide on Hair Growth. Annals of Dermatology. 2016;28(4):438443. doi: 10.5021/ad.2016.28.4.438.

[10] Gazitaeva ZI, Drobintseva AO, Chung Y, Polyakova VO, Kvetnoy IM. Cosmeceutical product consisting of biomimetic peptides: antiaging effects in vivo and in vitro. Clinical, Cosmetic and InvestigationalDermatology. 2017;10:11-16. doi:10.2147/CCID.S97573.

[11] Philp D, Nguyen M, Scheremeta B, St-Surin S, Villa AM, Orgel A, KleinmanHK,Elkin M. Thymosin beta 4 increases hair growth by activation of hair follicle stem cells. FASEB J. 2004 Feb;18(2):385-7. doi: 10.1096/fj.03-0244fje.

[12] Philp D, St-Surin S, Cha HJ, Moon HS, Kleinman HK, Elkin M. Thymosin beta 4 induces hair growth via stem cell migration and differentiation. Ann N Y Acad Sci. 2007 Sep;1112:95-103. doi: 10.1196/ annals.1415.009.

[13] Ito C, Saitoh Y, Fujita Y, Yamazaki Y, Imamura T, Oka S, Suzuki S. Decapeptidewith fibroblast growth factor (FGF)-5 partial sequence inhibits hair growthsuppressing activity of FGF-5. J Cell Physiol. 2003 Nov;197(2):272-83.

[14] Bassino E, Zanardi A, Gasparri F, Munaron L. Effects of the biomimetic peptide Sh-Polypeptide 9 (CG-VEGF) on cocultures of human hair follicle dermal papillacells and microvascularend othelial cells. ExpDermatol. 2016 Mar;25(3):237-9.doi: 10.1111/exd.12906.

[15] Schagen SK. Topical Peptide Treatments with Effective Anti-Aging Results. Cosmetics 2017, 4, 16; doi:10.3390/cosmetics4020016.

[16] Ito T, Ito N, Saathoff M, Bettermann A, Takigawa $\mathrm{M}$, Paus R. Interferon-gammais a potent inducer of catagen-like changes in cultured human anagen hair follicles. Br J Dermatol. 2005 Apr;152(4):623-31.

[17] Trink A, Sorbellini E, Bezzola P, Rodella L, Rezzani R, Ramot Y, Rinaldi F. A randomized, double- 
Evaluation of A Pool of Biomimetic Peptides on Human Hair Follicles: A Preclinical Study

blind, placebo- and active-controlled, half-head study to evaluate the effects of platelet-rich plasma on alopecia areata. Br J Dermatol. 2013 Sep;169(3):690-4. doi: 10.1111/bjd.12397.

[18] Fields K, Falla TJ, Rodan K, Bush L. Bioactive peptides: signaling the future. J CosmetDermatol. 2009 Mar;8(1):8-13. doi: 10.1111/j.14732165.2009.00416.x.
[19] Krause K, Foitzik K. Biology of the hair follicle: the basics. SeminCutan Med Surg. 2006 Mar; 25(1):2-10.

\section{Citation: Rodella Luigi Fabrizio, Rezzani Rita, Marzani Barbara, Pinto Daniela, Cocchi Marco, Sorbellini} Elisabetta, and Rinaldi Fabio. Evaluation of A Pool of Biomimetic Peptides on Human Hair Follicles: A Preclinical Study. Archives of Dermatology and Skin Care. 2019; 2(1): 11-17.

Copyright: (C) 2019 Rodella Luigi Fabrizio, Rezzani Rita, Marzani Barbara, Pinto Daniela, Cocchi Marco, Sorbellini Elisabetta, and Rinaldi Fabio. This is an open access article distributed under the Creative Commons Attribution License, which permits unrestricted use, distribution, and reproduction in any medium, provided the original work is properly cited. 